\title{
PRINCIPAL EIGENVALUE CHARACTERIZATION CONNECTED WITH STOCHASTIC PARTICLE MOTION IN A FINITE INTERVAL ${ }^{1}$
}

\author{
FETHI BIN MUHAMMAD BELGACEM \\ and \\ AHMED ABDULLATIF KARABALLI \\ Kuwait University \\ Department of Mathematics and Computer Science \\ P.O. Box 5969, Safat 130, Kuwait \\ E-mail:Belgacem@mcs.sci.kuniv.edu.kw,karabal@mcs.sci.kuniv.edu.kw
}

(Received January, 2002; Revised October, 2002 )

In this paper, we show that despite their distinction, both the Statonovich and Ito calculi lead to the same reactive Fokker-Planck equation:

$$
\frac{\partial p}{\partial t}-\frac{\partial}{\partial x}\left[D \frac{\partial p}{\partial x}-b p\right]=\lambda m p,
$$

describing stochastic dynamics of a particle moving under the influence of an indefinite potential $m(x, t)$, a drift $b(x, t)$, and a constant diffusion $D$. We treat the periodic-parabolic eigenvalue problem (1) for finite domains having absorbing barriers. We show that under conditions required by the maximum principle, the positive principal eigenvalue $\lambda^{*}$ (and the negative principal eigenvalue $\lambda_{*}$ ) is connected to the probability eigendensity function $p(x, t)$ by a Raleigh-Ritz like formulation. In the process, we establish the manner of effect of the drift and any inducing potential on the size of the principal eigenvalue. We show that the degree of convexity of the potential plays a major role in this regard.

Key words: Fokker-Planck Equation, Indefinite Potential, Principal Eigenvalue.

AMS subject classifications: 49J20, 49J40, 49K20, 65N25.

\section{Introduction}

In the absence of randomness, the directed motion of a particle on the real line due to an existing differentiable potential $B(x, t)$ (see [17]) is given by

$$
\frac{d x}{d t}=\frac{\partial B}{\partial x}=b(x, t) .
$$

The authors wish to acknowledge the support of the Kuwait University Research Administration (KURA) through grant: SM 04/01. 
The drift function $b(x, t)$ is then the velocity with which the particle travels, and in the event that $B$ does not vary with time, the motion of the particle is steady and satisfies the equation

$$
x(t)-B(x(t))=c .
$$

Assuming an additional random component for the motion of the particle without violating its continuity, equation (1.1) can be expanded to the Langevin equation:

$$
d x(t)=b(x, t)+s(x, t) \Phi(t) d t .
$$

Here, the function $\Phi(t)$ is rapidly fluctuating with time, having null statistical mean and Dirac $\delta$ variance function. The quantity $\Phi(t) d t$ is commonly known to be closely related to the Wiener process $w(t)$ (see [13]). The function $s(x, t)$ indicates the scale of randomness in the particle motion and is connected with the phenomenon of diffusion in the prescribed medium (see [9]). In fact, the diffusion coefficient of the medium $D(x, t)$ is known to be given by the relation:

$$
D(x, t)=\frac{s^{2}(x, t)}{2} .
$$

Clearly, $D(x, t)$ is a nonnegative function, and in this regard the Langevin equation may technically be called the stochastic drift-diffusion equation, and be written:

$$
d x(t)=b(x, t) d t+\sqrt{2 D(x, t)} d w(t) .
$$

The problem at hand is to estimate the influence of the drift $b(x, t)$ on the behavior of the particle motion described by the Langevin equation in a finite interval $\left[s_{1}, s_{2}\right]$ having absorbing ends. Assuming that the particle is subjected to a reactive potential $m(x, t)$ that changes sign in the interval $\left[s_{1}, s_{2}\right]$, we may ask how is the long term behavior of the particle affected if the drift is induced by $m(x, t)$. The standing conjecture (see $[2,5])$ is that for the reflecting barrier case a drift along $m_{x}$ should settle the particle mostly in areas where the potential $m$ is optimally positive. This is treated in [6]. Here, we only consider the absorbing barrier case.

\section{Stratonovich Versus Îto Integration}

Techniques for solving stochastic equations like (1.5) depart greatly from those for solving ODEs. In this regard, the authors of [11] state that: The stochastic nature of the Langevin equation allows an infinite number of possible trajectories for any given initial conditions. The probability of any one trajectory is governed by the statistical properties of the randomly fluctuating force. The value of equation (1.5) comes from our ability to integrate it when possible. Integrating (1.5) in the interval $\left[s_{1}, s_{2}\right]$ yields:

$$
x\left(s_{2}\right)-x\left(s_{1}\right)=\int_{s_{1}}^{s_{2}} b d t+\int_{s_{1}}^{s_{2}} \sqrt{2 D(x t)} d w(t) .
$$

The first integral in the previous equation is a Riemann-Stieltjes one, and it may be calculated by rules of ordinary calculus, while the second integral is stochastic in nature. Given a continuous, nonanticipating function $f(t)$ (see [13]), and a partition $s_{1}=t_{0}<t_{1}<\ldots<t_{n}=s_{2}$ of the interval $\left[s_{1}, s_{2}\right]$, we choose $\tau_{i}$ 's such that $t_{i-1} \leq \tau_{i} \leq t_{i}$ and compute the sequence of partial sums: 


$$
S_{n}=\sum_{i=1}^{n} f\left(\tau_{i}\right)\left[\omega\left(t_{i}\right)-\omega\left(t_{i-1}\right)\right]
$$

The value of $S_{n}$ depends greatly on how the $\tau_{i}$ 's are chosen and on the value of $f\left(\tau_{i}\right)$. If we set $\tau_{i}=\alpha t_{i-1}+(1-\alpha) t_{i}, 0 \leq \alpha \leq 1$, then $S_{n}=S_{n}\left(\alpha, f, s_{1}, s_{2}\right)$. The stochastic integral of $f$ may be defined in the following manner:

$$
I\left(\alpha, f, s_{1}, s_{2}\right)=\int_{s_{1}}^{s_{2}} f(t) d \omega(t)=m s \lim _{n} S_{n}\left(\alpha, f, s_{1}, s_{2}\right),
$$

where $m s$ means the limit in the mean square ( $L^{2}$ norm). The Ito stochastic integral is given by:

$$
I\left(1, f, s_{1}, s_{2}\right)=m s \bar{n} \lim _{\rightarrow} S_{n}\left(1, f, s_{1}, s_{2}\right) .
$$

The Stratonovich stochastic integral, on the other hand, is given by:

$$
I\left(\frac{1}{2}, f, s_{1}, s_{2}\right)=\oiint_{s_{1}}^{s_{2}} f(t) d \omega(t)=m s \underset{n}{\rightarrow} \lim _{\infty} S_{n}\left(\frac{1}{2}, f, s_{1}, s_{2}\right) .
$$

We remark that for $f(t) \equiv 1, I\left(1, f, s_{1}, s_{2}\right)=I\left(\frac{1}{2}, f, s_{1}, s_{2}\right)$, while

$$
I\left(1, \omega(t), s_{1}, s_{2}\right)=\frac{1}{2}\left[\omega^{2}(t)-t\right]_{s_{1}}^{s_{2}}
$$

and

$$
I\left(\frac{1}{2}, \omega(t), s_{1}, s_{2}\right)=\frac{1}{2}\left[\omega^{2}(t)\right]_{s_{1}}^{s_{2}} .
$$

Note that the Stratonovich integral looks more like the regular Riemann integral and the term $[t]_{s_{1}}^{s_{2}}=\left(s_{2}-s_{1}\right)$ that occurs in the Ito formulation does not show up in the Stratonovich model. While the Itto integral may be mathematically and technically more satisfactory, the Stratonovich quadrature may naturally be a better choice physically. Despite these discrepancies, it can be easily shown (see [1, 2]) that both approaches yield $(d \omega(t))^{\delta}=0$ if $\delta>2$, and $(d \omega(t))^{2}=d t$ in the mean square.

\section{Stratonovich Versus Îto Dynamical Model Formulation}

For developmental and historical reasons, the stochastic equation (1.5) describing a Markov process (See $[14,18]$ ) has become to be known as the Itto Differential Equation (IDE):

$$
d x(t)=b d t+\sqrt{2 D} d \omega(t)
$$

Another rival model to the IDE also describing particle motion due to drift and diffusion is given by the Stratonovich Differential Equation (SDE):

$$
d x(t)=\left[b-\frac{D_{x}}{2}\right] d t+\sqrt{2 D} d \omega(t) .
$$


Ito and Stratonovich formulations are identical if the diffusion $D$ is independent of the location. It is shown by the authors of [19] that both, the IDE (3.1) and the SDE (3.2), can lead to natural solutions. Here among other results, we develop their ideas further. The Ito change of variable formula is given by (see [1] for a proof):

$$
\begin{gathered}
d f(x(t))=\left[b f^{\prime}(x(t))+D f^{\prime \prime}(x(t))\right] d t+\sqrt{2 D} f^{\prime}(x(t)) d \omega(t) \\
=f^{\prime}(x(t)) d x(t)+D f^{\prime \prime}(x(t)) d t
\end{gathered}
$$

On the other hand, the Stratonovich chain rule is that of ordinary calculus as can be seen from the next result.

Theorem 3.1: For a differentiable function $f$ of $x(t)$ given by the $S D E(3.2)$,

$$
d f(x(t))=f^{\prime}(x(t)) d x(t) .
$$

Proof: We start with the SDE:

$$
d x=b^{S} d t+\sqrt{2 D} d \omega(t) .
$$

This equation may be converted into an IDE as follows:

$$
d x(t)=\left[b^{S}+\frac{D_{x}}{2}\right] d t+\sqrt{2 D} d \omega(t) .
$$

Now, letting $y=f(x)$ gives rise to the inverse $x=g(y)$, with

$$
\left(\frac{d g}{d y}\right)^{-1}=\frac{d y}{d g}=\frac{d f}{d x} .
$$

Furthermore, we have $D(x)=D(y)$ and $b^{S}(y)=b^{S}(x)$. After substitutions of these equalities, and manipulations of the IDE, we get:

$$
d y=\left[b^{S}\left(\frac{d g}{d y}\right)^{-1}+D_{y}\left(\frac{d g}{d y}\right)^{-2}-D \frac{d^{2} g}{d y^{2}}\left(\frac{d g}{d y}\right)^{-3}\right] d t+\sqrt{2 D}\left(\frac{d g}{d y}\right)^{-1} d \omega(t) .
$$

Now, we convert this IDE back to an SDE and obtain:

$$
d f(x)=d y=\left[b^{S} d t+\sqrt{2 D} d \omega(t)\right]\left(\frac{d g}{d y}\right)^{-1} .
$$

Using the previous defining relations, we recover the theorem conclusion.

It was shown (see $[1,6]$ ) that a Langevin particle subject to diffusion $D(x, t)$ and to drift $b(x, t)$, hence governed by the IDE (3.1), resulting in the probability density $p(x, t)$ evaluating the chances of the particle being at location $x$ at every time $t$, and a probability flux:

$$
J(x, t)=b(x, t) p(x, t)-\frac{\partial}{\partial x}[D(x, t) p(x, t)] .
$$

It must satisfy the Fokker-Planck Equation (FPE):

$$
\frac{\partial p}{\partial t}=-\frac{\partial}{\partial} J=\frac{\partial}{\partial x}\left[\frac{\partial}{\partial x}(D p)-b p\right] .
$$

The next result establishes the correct form of the FPE due to a Stratonovich drift. 
Theorem 3.2: Assuming that the Langevin particle moves under the influence of a Stratonovich drift $b^{S}(x, t)$ yields the corresponding FPE:

$$
\frac{\partial p}{\partial t}=\frac{\partial}{\partial x}\left[\sqrt{D} \frac{\partial}{\partial x}(\sqrt{D} p)-b^{S} p\right] .
$$

Proof: Since $b^{S}=b-\frac{D_{x}}{2}=b-s s_{x}$, where $b$ is the Itto drift, we see that the FPE (3.10) can lead to

$$
\frac{\partial p}{\partial t}=\frac{\partial}{\partial x}\left[\frac{\partial}{\partial x}(D p)-b p\right]=\frac{\partial}{\partial x}\left[D \frac{\partial p}{\partial x}+\frac{D_{x}}{2} p-\left(b-\frac{D_{x}}{2}\right) p\right] .
$$

Exchanging $\frac{s^{2}}{2}$ for $D$ we get:

$$
\frac{\partial p}{\partial t}=\frac{\partial}{\partial x}\left[\frac{s^{2}}{2} \frac{\partial p}{\partial x}+\frac{s s_{x}}{2} p-b^{S} p\right]=\left[\frac{s}{\sqrt{2}} \frac{\partial}{\partial x}\left(\frac{s}{\sqrt{2}} p\right)-b^{S} p\right] .
$$

Substituting $\sqrt{D}$ for $\frac{s}{\sqrt{2}}$ in equation (3.13) we get the Stratonovich FPE (3.11).

Clearly, both formulations coincide if diffusion is isotropic; $D(x)=D=$ constant. Moreover, both equations (3.10) and (3.11) are valid in the absence of reactions. In the next section, we treat reactive versions of the FPEs with constant diffusion. In the absence of drift, equations (3.1) and (3.5) lead to the heat equation versions of (3.10) and (3.11) (see [10, 12, 18]). Introduction of drift into the FPEs helped explain many cellular dynamic phenomena (see, for instance, [3, 4, 9, 21]).

\section{Indefinite Reactions Effects on the Dynamics of a Langevin Particle}

In this section, we treat the isotropic case of a Langevin particle subject to a $T$-periodic drift $b(x, t)$ and a $T$-periodic indefinite reactive potential $m(x, t)$. We seek a probability density function $p(x, t)$ to the $T$-periodic parabolic eigenvalue problem

$$
\frac{\partial p}{\partial t}-\frac{\partial}{\partial x}\left[D \frac{\partial p}{\partial x}-b p\right]=\lambda m p \text { in }\left(s_{1}, s_{2}\right) \times[0, T],
$$

with initial density $p_{0}=p(x, 0)$, such that at all times, the given data at the boundary points $s_{1}$ and $s_{2}$ is $p=0$. This means that we have Dirichlet conditions at

the endpoints. The driftless problem with reflecting barriers at the endpoints $\left(\frac{\partial p}{\partial x}=0\right)$ was treated in [8]. Further developments including drift can be found in [3,

$5,6,16]$. Here, however, we focus on the Dirichlet case.

The recent results of [7] imply that under Dirichlet conditions, provided $\left.\int_{0}^{T} \max _{\left[s, s_{2}\right]}(m(x, t) d t)\right)>0$, problem (4.1) admits a principal eigenvalue $\lambda^{*}>0$ having a positive $T$-periodic density $p(x, t)$. An easy consequence of the previous statement shows that provided $\left.\int_{0}^{T} \min _{\left[s_{1}, s_{2}\right]}(m(x, t) d t)\right)<0$, problem (4.1) admits a negative principal eigenvalue $\lambda_{*}$ having a $T$-periodicity density $q(x, t)$. This can be easily seen by realizing that in problem (4.1), the quantity $\lambda m p=(-\lambda)(-m) p$. Both $p$ and $q$ are in $C^{2+\theta, 1+\frac{\theta}{2}}\left(\left[s_{1}, s_{2}\right] \times \mathbb{R}\right)$ if $b$ and $m$ are in $C^{1+\theta, 1+\frac{\theta}{2}}\left(\left[s_{1}, s_{2}\right] \times \mathbb{R}\right)$.

The next few results connect $\lambda^{*}$ and $p$ to the coefficients of the eigenvalue problem.

Theorem 4.1: Let $m$ and $b$ be T-periodic in $t$, and let $b(x, t)$ be differentiable with respect to $x$, then th positive principal eigenvalue $\lambda^{*}$ of problem (4.1) subject to Dirichlet 
conditions $\left(p\left(s_{2}, t\right)=p\left(s_{1}, t\right)=0\right.$ is connected to the corresponding eigendensity function $p(x, t)$ by the following

$$
\lambda^{*} \int_{0}^{T} \int_{s_{1}}^{s_{2}} m p^{2} d x d t=D \int_{0}^{T} \int_{s_{1}}^{s_{2}}\left(p_{x}\right)^{2} d x d t+\int_{0}^{T} \int_{s_{1}}^{s_{2}} \frac{b_{x}}{2} p^{2} d x d t
$$

Proof: Multiplying problem (4.1) by $p$ and integrating over $\left[s_{1}, s_{2}\right]$, we get:

$$
\begin{gathered}
\lambda \int_{s_{1}}^{s_{2}} m p^{2} d x=\int_{s_{1}}^{s_{2}} J_{x} p d x+\int_{s_{1}}^{s_{2}} \frac{\partial p}{\partial t} d x \\
=\int_{s_{1}}^{s_{2}}[J p]_{x} d x-\int_{s_{1}}^{s_{2}} J p_{x} d x+\int_{s_{1}}^{s_{2}} \frac{\partial p}{\partial t} d x .
\end{gathered}
$$

Using Dirichlet boundary conditions, the first integral on the right-hand side vanishes, and the second integral yields:

$$
-\int_{s_{1}}^{s_{2}} J p_{x} d x=D \int_{s_{1}}^{s_{2}}\left(p_{x}\right)^{2} d x+\int_{s_{1}}^{s_{2}} \frac{b_{x}}{2} p^{2} d x
$$

Therefore, we have

$$
\lambda \int_{s_{1}}^{s_{2}} m p^{2} d x=D \int_{s_{1}}^{s_{2}}\left(p_{x}\right)^{2} d x+\int_{s_{1}}^{s_{2}} \frac{b_{x}}{2} p^{2} d x+\int_{s_{1}}^{s_{2}} \frac{\partial p}{\partial t} d x
$$

Integrating equation (4.5) on interval $[0, T]$, we obtain:

$$
\int_{0}^{T} \int_{s_{1}}^{s_{2}} \frac{\partial p}{\partial t} d x d t=\int_{s_{1}}^{s_{2}} \int_{0}^{T} \frac{\partial p}{\partial t} d t d x=\int_{s_{1}}^{s_{2}}[p(x, t)]_{0}^{T} d x \equiv 0
$$

Since $p(x, t)$ is $T$-periodic, we get:

$$
\lambda \int_{0}^{T} \int_{s_{1}}^{s_{2}} m p^{2} d x d t=D \int_{0}^{T} \int_{s_{1}}^{s_{2}}\left(p_{x}\right)^{2} d x d t+\int_{0}^{T} \int_{s_{1}}^{s_{2}} \frac{b_{x}}{2} p^{2} d x d t .
$$

So that if $\lambda=\lambda^{*}, p$ is the corresponding eigendensity. 
Remarks: Similarly if $\lambda=\lambda_{*}$, then $p=q$. Also, note that if we denote $\lambda^{*}=$ $\lambda(D, b, m)$, we see that $\lambda(D, b=$ constant, $m)=\lambda(D, 0, m)$. In other words, a constant drift in $\left[s_{1}, s_{2}\right]$ has no effect on the value of the eigenvalue $\lambda^{*}$ (nor on the value of $\lambda_{*}$ ). This is due to the fact that $\lambda^{*}$ is the smallest nonnegative eigenvalue of problem (4.1).

In the next results, we consider the situation where for real $\alpha$,

$$
b=\alpha \frac{\partial m}{\partial x} .
$$

Corollary 4.2: Let $m$ and $b$ in Theorem 4.1 be related through equation (4.8). Then:

$$
\lambda^{*} \int_{0}^{T} \int_{s_{1}}^{s_{2}} m p^{2} d x d t=D \int_{0}^{T} \int_{s_{1}}^{s_{2}}\left(p_{x}\right)^{2} d x d t+\frac{\alpha}{2} \int_{0}^{T} \int_{s_{1}}^{s_{2}} m_{x x} p^{2} d x d t
$$

Proof: Clearly if $b$ is related to $m$ through relation (4.8), then:

$$
b_{x}=\alpha \frac{\partial^{2} m}{\partial x^{2}} .
$$

Substituting this in equation (4.7) yields the desired result.

Equation (4.9) shows that the principal eigenvalue $\lambda^{*}$ is influenced by the degree of concavity or convexity of the potential $m(x, t)$. The next result is concerned with the influence of the drift $b$ and weight $m$ on the eigenvalue $\lambda^{*}$ (and $\lambda_{*}$ ) in the steady case (long term behavior).

Corollary 4.3: In the steady state case with $b=b(x), m=m(x)$, we have $p=p(x)$ and

$$
\lambda^{*} \int_{s_{1}}^{s_{2}} m p^{2} d x=D \int_{s_{1}}^{s_{2}}\left(p_{x}\right)^{2} d x+\int_{s_{1}}^{s_{2}} \frac{b_{x}}{2} p^{2} d x
$$

Furthermore, if $b$ and $m$ are related via equation (4.8), then

$$
\lambda^{*} \int_{s_{1}}^{s_{2}} m p^{2} d x=D \int_{s_{1}}^{s_{2}}\left(p_{x}\right)^{2} d x+\frac{\alpha}{2} \int_{s_{1}}^{s_{2}} m_{x x} p^{2} d x
$$

Proof: Equations (4.11) and (4.12) are obtained from (4.2) and (4.9) respectively, by the obvious substitution, for the Dirichlet eigenvalue problem in $\left(s_{1}, s_{2}\right)$ :

$$
-\frac{\partial}{\partial x}\left[D \frac{\partial p}{\partial x}-b p\right]=\lambda m p
$$

considered periodic-parabolic for every period $T>0$.

\section{References}

[1] Belgacem, F., Diffusion and drift models for population dispersal from stochastic and continuum views, IJAM 5:1 (2001), 85-106.

[2] Belgacem, F., Boundary value problem with indefinite weight and applications, IJPNA in Eng. Sys. 10:2 (1999), 51-58. 
[3] Belgacem, F., Elliptic Boundary Value Problems with Indefinite Weights: Variational Formulations of the Principal Eigenvalue and Applications, Pitman Research Notes in Mathematics Series 368, Addison-Wesley-Longman 1997.

[4] Belgacem, F., Modeling Platelet Transport in Flowing Blood, Master thesis in Biomedical Engineering, University of Miami, Coral Gables, FL 1990.

[5] Belgacem, F. and Cosner, C., The effects of disposal along environmental gradients on the dynamics of populations in heterogeneous environments, Canadian Appl. Math. Qtrly. J. 3:4 (1995), 379-397.

[6] Belgacem, F. and Karaballi, A.A. The motion of a particle in a finite interval subject to an indefinite potential and the stochastic Cosner conjecture, Dyn. of Cont., Disc. and Imp. Systems, Series B 9 (2002), 139-151.

[7] Belgacem, F. and Smaoui, N., Interaction of parabolic connective diffusion equations and Navier-Stokes equations connected with population dispersal, Comm. on Appl. Nonl. Anal. 8:3 (2001), 47-67..

[8] Brown, K.J. and Lin, S.S., Bifurcation and stability results for an equation arising in population genetics, Anna. di Math. Pura y Appl. 128 (1981), 375-387.

[9] Eckstein, E.C. and Belgacem, F., Model of platelet transport in flowing blood with drift and diffusion terms, Biophy. J. 60 (1991), 53-69.

[10] Einstein, A., Investigations on the Theory of Brownian Movement, Dover Publications Inc., New York 1956.

[11] Ermak, D.L. and Buckholz, H., Numerical integration of the Langevin equation: Monte Carlo simulation, J. of Comp. Phy. 25 (1980), 169-182.

[12] Feller, W., Diffusion processes in one-dimension, Trans. Amer. Soc. 77:1 (1945), 1-31.

[13] Gardiner, C.W., Handbook of Stochastic Methods for Physics, Chemistry, and Natural Sciences, Springer-Verlag, New York 1988.

[14] Gillespie, T.D., Markov Processes: An Introduction for Physical Scientists, Academic Press Inc., New York 1992.

[15] Hess, P., Periodic-Parabolic Boundary Value Problems and Positivity, Pitman, New York 1991.

[16] Holland, C.J. A new energy characterization of the smallest eigenvalue of the Schrodinger equation, Comm. on Pure and Appl. Math. XXX (1977), 755-765.

[17] Karaballi, A.A., On the ejection of solutions in a central force field, Celestial Mech. $\mathbf{4 1}$ (1988), 323-332.

[18] Karatzas, I. and Shreve, S., Brownian Motion and Stochastic Calculus, SpringerVerlag, New York 1989.

[19] Krasnosel'skii, M.A. and Pokrovskii, A.V., Natural solutions of stochastic differential equations, Soviet Math. Dokl. 19:3 (1978), 578-582.

[20] Manes, A. and Micheletti, A.M. Un extension della teoria variazionale classica degli autovalori per operatori ellittiei del secondo ordine, Boll. UMI 7 (1973), 285-301.

[21] Watkins, J.C. and Woessner, B., Diffusion models for chemotaxis: A statistical analysis of noninteractive unicellular movement, Math. Biosci. 104 (1991), 271-303. 


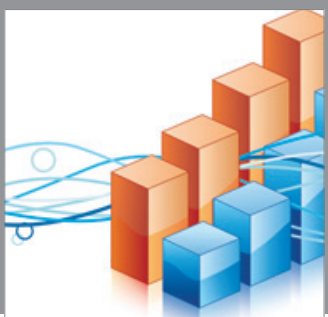

Advances in

Operations Research

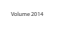

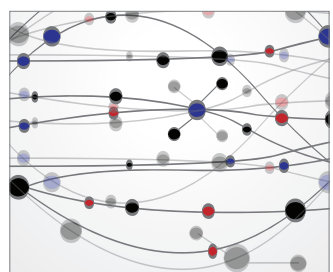

\section{The Scientific} World Journal
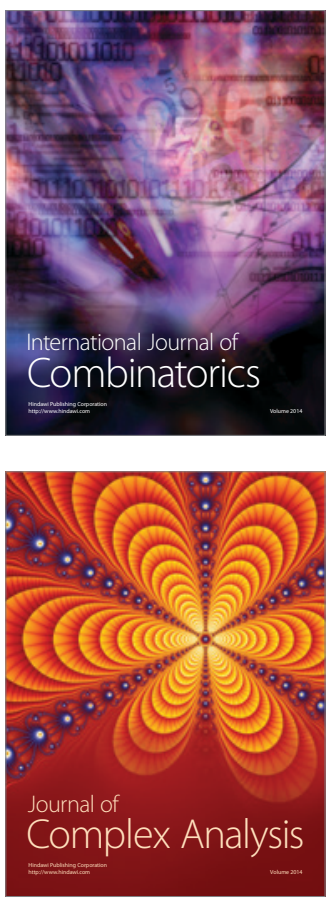

International Journal of

Mathematics and

Mathematical

Sciences
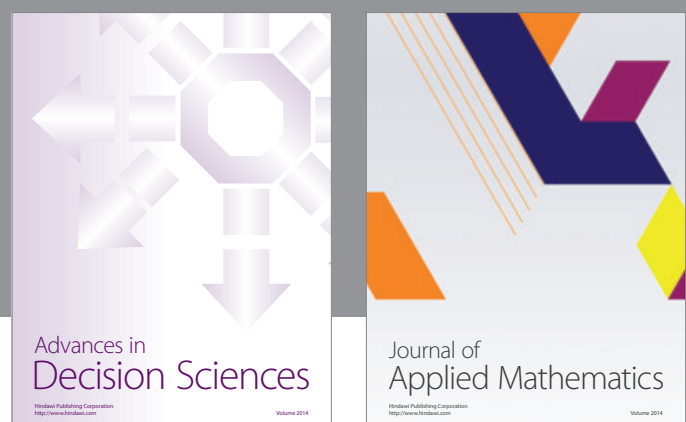

Journal of

Applied Mathematics
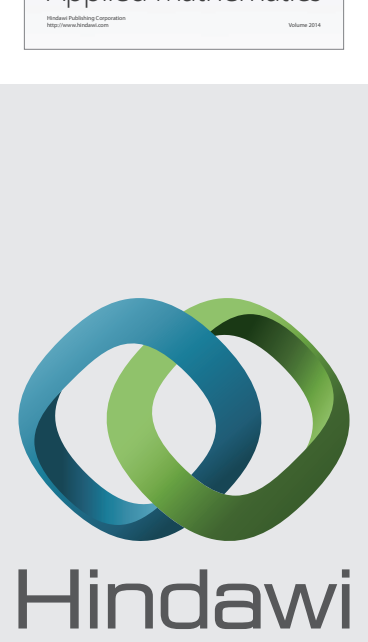

Submit your manuscripts at http://www.hindawi.com
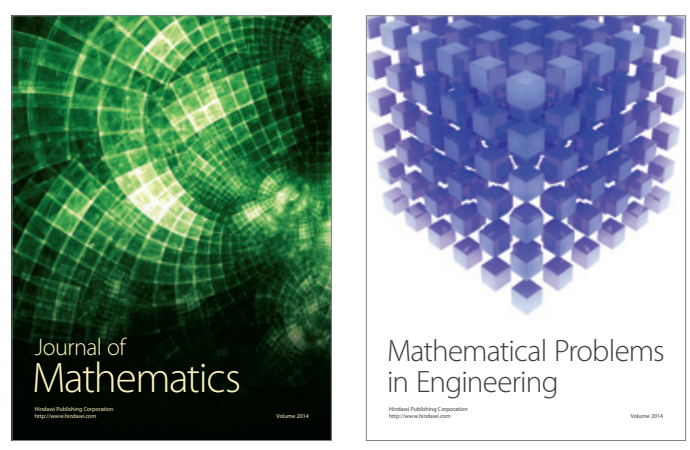

Mathematical Problems in Engineering
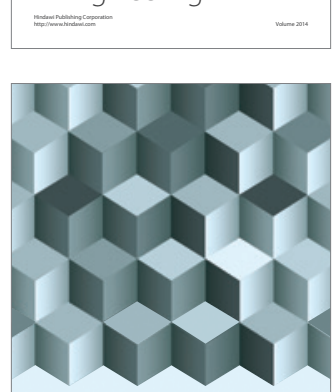

Journal of

Function Spaces
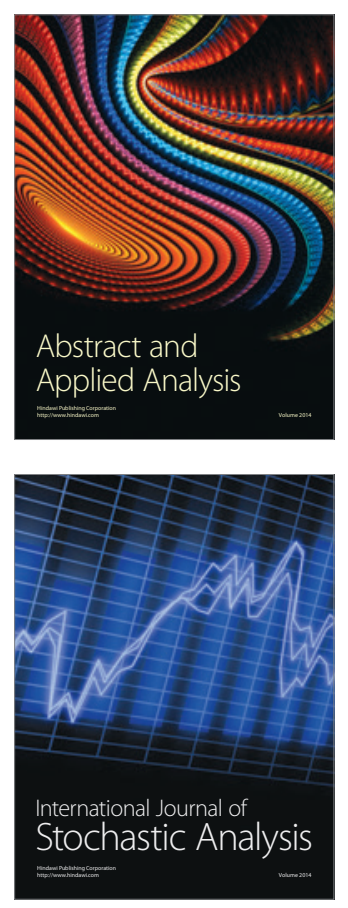

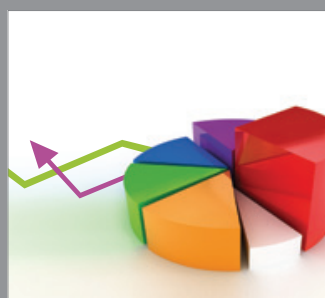

ournal of

Probability and Statistics

Promensencen
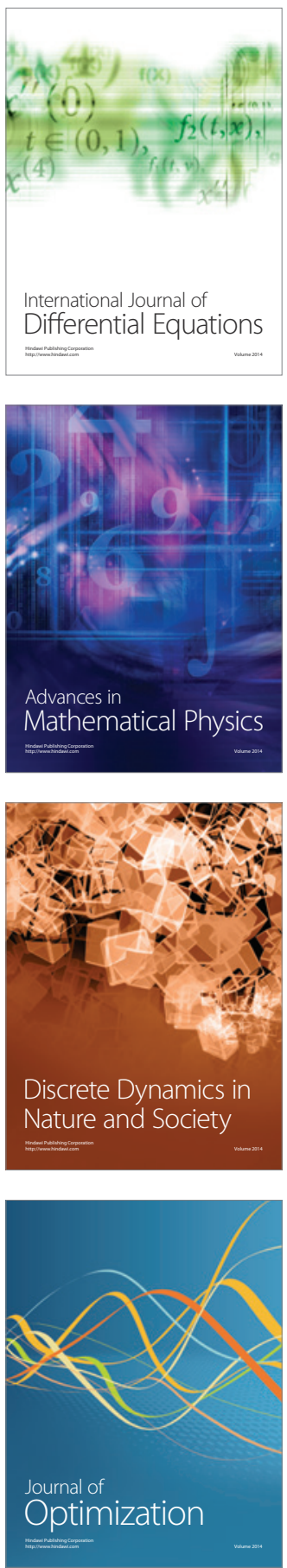